\title{
SMAI - Sistema Móvel de Assistência ao Idoso
}

\author{
Matheus Costa Stutzel ${ }^{1}$, Michel Filippo ${ }^{1}$, Luciana Branco da Motta $^{3}$, Alexandre \\ Sztajnberg ${ }^{1,2,4}$, André Brittes ${ }^{3}$
}

\author{
Universidade do Estado do Rio de Janeiro, Rio de Janeiro, RJ \\ ${ }^{1}$ Bacharelado em Ciência da Computação - Instituto de Matemática e Estatística \\ ${ }^{2}$ Pós-Graduação em Ciências Computacionais - Instituto de Matemática e Estatística \\ ${ }^{3}$ Núcleo de Apoio ao Idoso - Universidade Aberta da Terceira Idade \\ ${ }^{4}$ Pós-Graduação em Eletrônica - Faculdade de Engenharia \\ matheusstutzel@yahoo.com.br, michelpf13@gmail.com, lubmotta@gmail.com, \\ alexszt@ime.uerj.br, brites84@gmail.com
}

\begin{abstract}
SMAI uses Android applications as the infrastructure to monitor elderly patients with chronic degenerative disease that present functional loss. The objectives are: (i) make the caregiver communication with the health team more agile; (ii) reduce caregiver stress; (iii) provide the health team information about the patient's condition more often in an organized manner, facilitating decision-making. Applications are developed under the guidance of the health team and are being assessed in a clinical trial with $30+30$ patients accompanied by NAI/UERJ.
\end{abstract}

Resumo. O SMAI utiliza aplicações Android como infraestrutura de monitoramento de pacientes idosos com doença crônico-degenerativa que apresentem perda funcional. Os objetivos são: (i) tornar a comunicação do cuidador com a equipe de saúde mais ágil; (ii) reduzir o estresse do cuidador; (iii) prover à equipe de saúde informações sobre o estado do paciente com maior frequência, de forma organizada, facilitando a tomada de decisões. As aplicações são desenvolvidas segundo a orientação da equipe de saúde e estão sendo avaliadas em um ensaio clínico com $30+30$ pacientes acompanhados pelo NAI/UERJ.

\section{Introdução}

As patologias crônicas têm potencial para causar incapacidades, somando prejuízos na autonomia e independência para a vida diária e aumentando a necessidade de cuidados prolongados prestados por familiares, amigos e comunidade [MinS, 2006]. Estima-se que entre 2010 e 2050 o número de idosos que necessitam de cuidados, em todo o mundo, quase triplicará de 101 para 277 milhões [Prince, 2013].

A família é a principal forma de suporte social disponível para o cuidado. A legislação e as políticas públicas afirmam e a própria sociedade acredita que os idosos devem ser cuidados por sua família [Caldas, 2003]. No seio familiar, a presença do idoso dependente implica em sobrecarga emocional, física e financeira [UNFPA, 2012]. Desta forma, é importante desenvolver ações que permitam que a equipe de saúde apoie os responsáveis pelo cuidado, aliviando o estresse de cuidar.

Na Universidade Aberta da Terceria Idada da UERJ (UnATI/UERJ), o Núcleo de Atenção ao Idoso (NAI) oferece um serviço de monitoramento contínuo para pacientes idosos com vários estágios de desordem neurocognitiva (demência). Uma 
equipe de saúde multidisciplinar acompanha 250 pacientes e seus cuidadores. O serviço é gratuito. Consultas médicas ambulatoriais são agendadas periodicamente para cada paciente, acompanhados por seus cuidadores. São seguidos protocolos e procedimentos estabelecidos, e as recomendações são enfatizadas com os cuidadores em situações de emergência. O serviço é oferecido tendo em conta as características do grupo de pacientes monitorados e seus cuidadores: (i) o cuidador é geralmente um membro da família e constantemente está estressado, (ii) a família tem, em média, baixo rendimento e a maioria reside em bairros periféricos e (iii) necessitam de transporte público para chegar ao NAI. Assim, cada encontro impõe sacrifício para o cuidador e paciente.

A tecnologia da informação tem despontado como recurso de apoio, atuando como facilitador da comunicação e monitoramento. $O$ grande avanço nas áreas de dispositivos de hardware e telecomunicações pavimentou o caminho para aplicações de telemedicina [Wootton, 2006]. Entre estas, as aplicações de telessaúde, onde dispositivos de monitoramento permitem o telemonitoramento e telediagnóstico vem sendo pesquisadas. Assim, a telemedicina pode integrar a rede de cuidado do idoso dependente, para complementar o atendimento presencial pela equipe de saúde.

Este artigo apresenta o SMAI - Sistema Móvel de Assistência ao Idoso, cujos objetivos são (i) tornar a comunicação do cuidador com a equipe de saúde mais ágil; (ii) reduzir o estresse do cuidador; (iii) facilitar o cuidado e suporte do idoso nas suas atividades de vida diária; (iv) prover à equipe de saúde informações sobre o estado do paciente com maior frequência, de forma organizada, facilitando a tomada de decisões. Além disso, são avaliados os impactos do uso do sistema na melhoria da qualidade de vida dos pacientes e cuidadores, nas intercorrências e na aderência aos tratamentos.

Para alcançar tais objetivos foi desenvolvido um sistema composto de 2 aplicações: uma dedicada ao profissional de saúde e otimizada para a utilização em um tablet, e a outra desenvolvida para smartphone, dedicada ao cuidador ou paciente. Elas foram projetadas para o contexto do grupo de pacientes e cuidadores monitorados pelo NAI, considerando as características e os condicionalismos deste grupo, as atuais práticas de saúde adotadas e a experiência da equipe no cuidado deste grupo, portanto as aplicações possuem interfaces desenvolvidas de modo a facilitar a visualização dos dados gerados pelos pacientes, bem como simplificar a entrada de novas informações no sistema.

O restante do texto está estruturado da seguinte forma. A Seção 2 apresenta trabalhos relacionados que consideram tecnologia da informação aplicada à telessaúde. A Seção 3 apresenta o contexto do projeto e o estudo preliminar realizado para nortear a definição do sistema. A Seção 4 apresenta uma visão geral do sistema e a Seção 5 aborda o protótipo desenvolvido e alguns detalhes da implementação. A Seção 6 discute uma primeira avaliação realizada e a Seção 7 traz as últimas considerações.

\section{Trabalhos relacionados e experiências anteriores}

O uso da telemedicina no controle de doenças crônicas tem sido discutido nos últimos anos, demostrando benefícios para o paciente e para os sistemas de saúde [Dorsey, 2013], [Celler, 2006], [Jaglal, 2013], [Finkelstein, 2006]. Alguns deles se concentram em sistemas de notificação e lembretes aos pacientes, com o objetivo de melhorar a aderência ao tratamento [Fisher, 2006], [Keranen, 2013]. 
O uso de soluções móveis e tecnologia da informação na área da telemedicina no Brasil está se consolidando. Considerando aplicações de telessaúde, algumas propostas já podem ser elencadas, como [Sanches, 2012].

Em [Nicholas, 2007] são avaliados vários aspectos da tecnologia para e-Health, incluindo o impacto para o paciente, para o médico e para os serviços de saúde já estabelecidos. Um dos problemas recorrentes é o abandono do uso do sistema, por várias razões: falta de incentivo, falta de interesse e a não percepção de benefícios.

A revisão apresentada em [Mosa, 2012] classifica aplicações para smartphones apresentadas em 2894 artigos. A maior parte das aplicações está relacionada à diagnóstico de doenças ou calculadoras médicas. Apenas 15 das aplicações pesquisadas estão relacionadas ao monitoramento de pacientes com doenças crônicas. Destas 15 aplicações poucas se preocupam em tornar lúdico o uso da aplicação. No SMAI procuramos adicionar aspectos lúdicos e características de gamificação ao aplicativo destinado aos pacientes/cuidadores como forma de atrair o usuário ao uso contínuo do sistema (mais detalhes são discutidos em [Stutzel, 2016]).

\section{Preparação}

O projeto do SMAI envolve duas equipes: o LCC - Laboratório de Ciência da Computação e pelo NAI - Núcleo de Atenção ao Idoso/ UnATI, ambos da UERJ. O público alvo do sistema é composto de idosos, com algum grau de dependência, e seus cuidadores. A equipe do NAI tem experiência no monitoramento e acompanhamento deste grupo empregando os procedimentos e as práticas disponíveis.

O SMAI é proposto com foco em aplicações simples, para serem utilizadas em um equipamento do tipo smartphone que já faz parte do dia-a-dia do paciente ou cuidador. $\mathrm{O}$ entendimento é o de que o uso de um aparelho de telefonia celular não introduza mudanças significativas na rotina das pessoas.

$\mathrm{O}$ acesso à Internet tornou-se ubíquo e economicamente mais acessível. Equipamentos que permitem acesso à Internet também se tomaram mais baratos. Ainda assim, devido ao baixo poder aquisitivo da maioria dos pacientes e cuidadores monitorados, para a avaliação do sistema é fornecido um smartphone com acesso à Internet para transmissão de dados e recebimento de alertas para cada paciente. Como preparação para o desenvolvimento do SMAI, foi realizado um levantamento preliminar sobre o uso de recursos de computação e telefonia móvel, aplicando-se um questionário ao grupo acompanhado pelo NAI. Ao todo, 42 (quarenta e dois) pacientes ou seus acompanhantes, responderam às questões do formulário. Do total dos entrevistados:

- $76.60 \%$ têm computador e $93.62 \%$ celular. $72.34 \%$ sabem enviar SMS (torpedo);

- $80.85 \%$ fazem uso de correio eletrônico e/ou redes sociais como o Facebook;

- $72.34 \%$ tem acesso à Internet em casa, sendo que destes $73 \%$ acha o acesso bom;

- $86 \%$ acessa a Internet algumas vezes por semana, sendo que $40 \%$ todos os dias.

Os resultados obtidos constituíram importante informação para direcionar a abordagem proposta para o SMAI. O uso de um aparelho celular é comum para a maioria dos pacientes, familiares ou cuidadores. Destes, $25.53 \%$ já utilizam o sistema Android [Google, 2003]. Com base nestas informações o sistema Android foi adotado. 


\section{SMAI - Sistema Móvel de Assistência ao Idoso}

O SMAI é composto de duas aplicações: paciente/cuidador e médico, e um serviço na nuvem. Os usuários têm acesso ao sistema através de um aparelho do tipo smartphone ou tablet, tanto em suas residências, como quando em mobilidade, para realizar seus afazeres fora de casa. A Figura 1 apresenta uma visão geral do SMAI.

Nos aparelhos smartphone uma aplicação ( $a p p$ ) permite ao paciente, ou ao cuidador/familiar enviar e receber informações e notificações da equipe de saúde:

- lembretes sobre uso de medicação e consultas agendadas;

- informações contendo medidas fisiológicas como a pressão arterial;

- relatório diário com informações sobre o comportamento, rotina alimentar, evacuação e micção do paciente; e relatório semanal sobre o próprio cuidador;

- recebimento de mensagens de suporte ao cuidador nas orientações de cuidado;

- envio periódico da localização do paciente, usando uma combinação de possibilidades como o GPS e sistemas de localização baseado na rede.

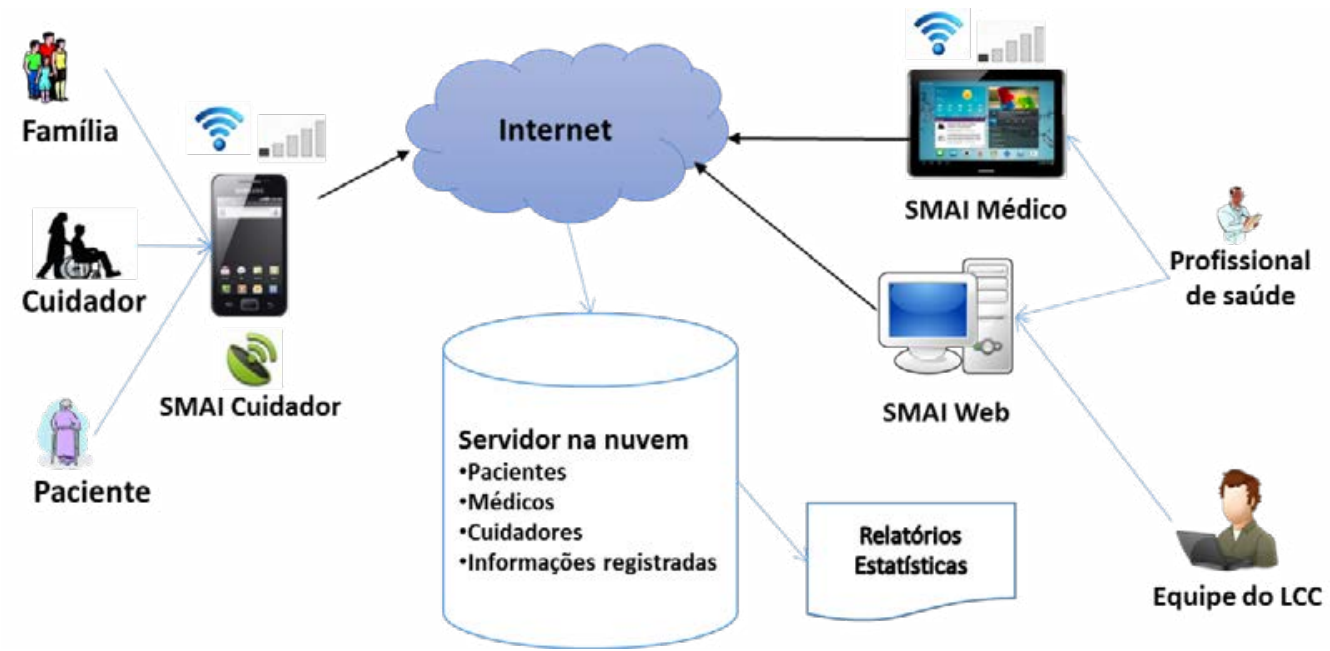

Figura 1. Esquema geral do SMAI

A aplicação utilizada pelo cuidador/familiar tem o objetivo de auxiliar seu dia a dia e facilitar o envio de informações para o NAI. A aplicação quando utilizada pelo paciente tem um número reduzido de opções, cujo objetivo é receber deste paciente informações sobre seu estado geral, mantendo uma conexão "virtual" com o NAI.

Os profissionais da saúde têm acesso ao sistema através de um tablet onde uma aplicação permite o monitoramento e a comunicação com o grupo de pacientes, com uma visualização estruturada das informações. Entre as funções estão:

- visualizar alarmes e informações atualizadas dos pacientes, em grupo;

- visualizar detalhes de cada paciente, incluindo a sua localização atual e dos últimos dias, permitindo verificar sua mobilidade;

- envio de agendamento de consulta e lembretes de medicamentos, além de mensagens para usuários individuais ou grupos de pacientes;

- visualizar os relatórios relacionados aos pacientes e o relatório do cuidador.

Todas as informações são enviadas para um servidor de aplicação na nuvem que oferece um conjunto de serviços específico de uma API. Da mesma forma, deste serviço 
são recebidas as mensagens, notificações e alarmes. Este serviço possui os requisitos de disponibilidade e segurança necessários para aplicações de telessaúde.

Privacidade e Segurança. A comunicação de cada ator do sistema, equipe de saúde e cuidadores, é feita a partir de uma identificação única no serviço na nuvem, permitindo a autenticação, o controle de acesso e privacidade. Antes de serem transmitidos, os dados são criptografados. Com isso é garantida a autenticidade e confidencialidade da informação sem exigir a identificação do usuário a cada acesso. $\mathrm{O}$ acesso aos dados dos pacientes é controlado por mecanismos de permissão e compartilhamento autorizado. Os agendamentos, lembretes de medicações e notificações para os pacientes tem associada a informação do profissional de saúde responsável.

Transmissão e Tolerância a Falhas. Os dados de todas as aplicações são transmitidos e recebidos pela Internet. $\mathrm{Na}$ ausência de conexão com a internet, o SMAI disponibiliza a visualização das informações já carregadas e os dados gerados localmente pelo usuário são persistidos localmente, até que uma conexão seja estabelecida.

\section{Protótipo}

As funcionalidades, painéis de informação e notificações foram implementadas de acordo com a orientação e requisitos da equipe de saúde do NAI, seguindo as práticas e padrões de projeto recomendados pela Google. A Figura 2 apresenta a estrutura geral adotada para as duas aplicações. Cada módulo herda um conjunto de classes *Activity, que transmitem/recebem os dados, em XML, criptografados, para/do serviço da nuvem.

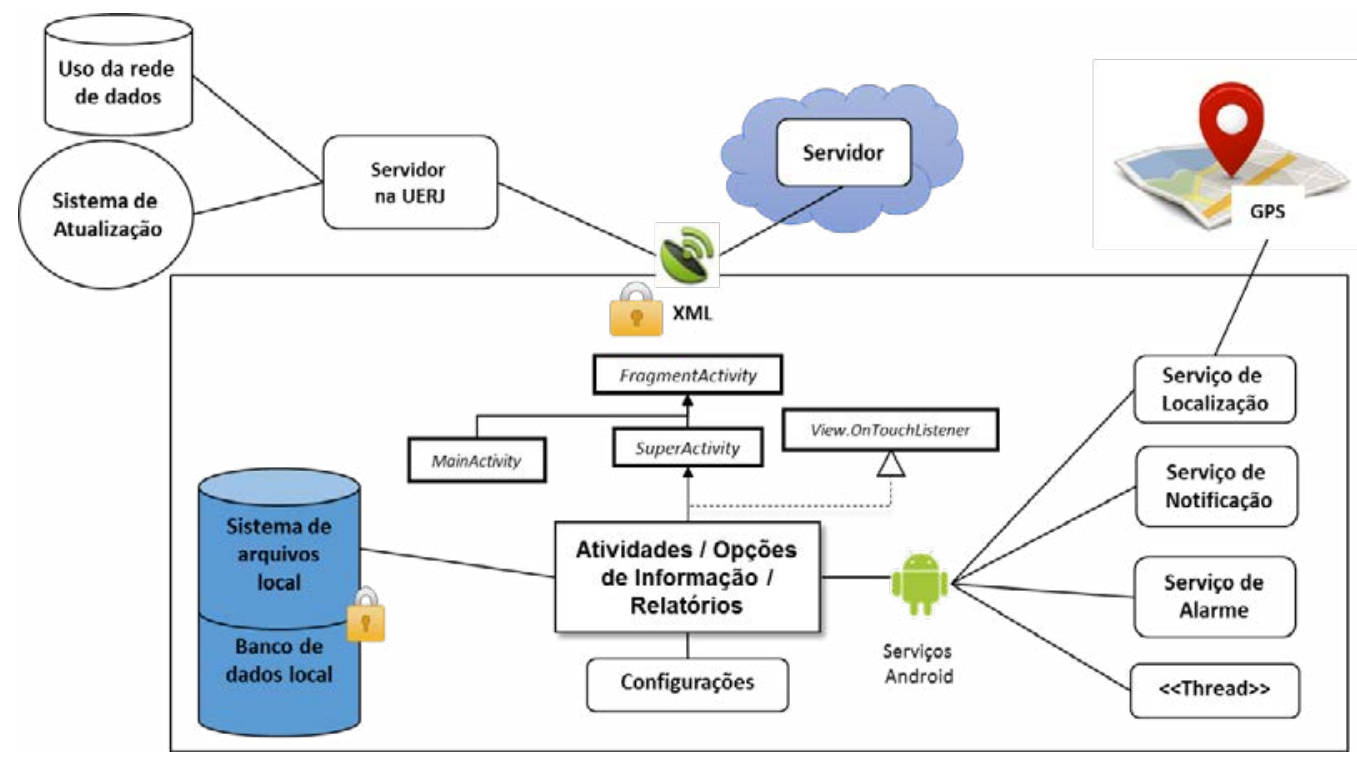

Figura 2. Estrutura do SMAI

As notificações no SMAI utilizam um mecanismo especialmente adaptado que permite, por exemplo, criar lembretes que demandam do cuidador uma confirmação e que não se acumulam evitando sobrecarga para o cuidador. Quando geradas, as notificações são acompanhadas de um alerta sonoro e visual no dispositivo.

Todos os dados gerados são salvos imediatamente na memória interna do equipamento para que não haja perda de dados. Após esse processo, é iniciado o envio para o servidor na nuvem. O procedimento de transmissão criptografa os dados a serem enviados e assim que o aplicativo recebe a confirmação a cópia local é apagada (no caso 
do smartphone), ou persistida no banco de dados local (tablet). Em caso de erro na transmissão, o processo é reiniciado após um tempo, que varia de acordo com o erro.

SMAI Cuidador. A aplicação do cuidador possui um menu principal, com módulos de entrada de informações (Figura 3). Ao ser carregado, o programa inicia todas as atividades de plano de fundo, como por exemplo, o envio da localização do paciente e a verificação de atualizações. A Figura 3 apresenta exemplos de entrada de dados de Dor, Pressão Arterial e Temperatura. Alguns módulos são cientes de contexto, acionando ações específicas. Por exemplo, se a temperatura ultrapassar $37,8^{\circ}$ é solicitada a informação sobre tosse.
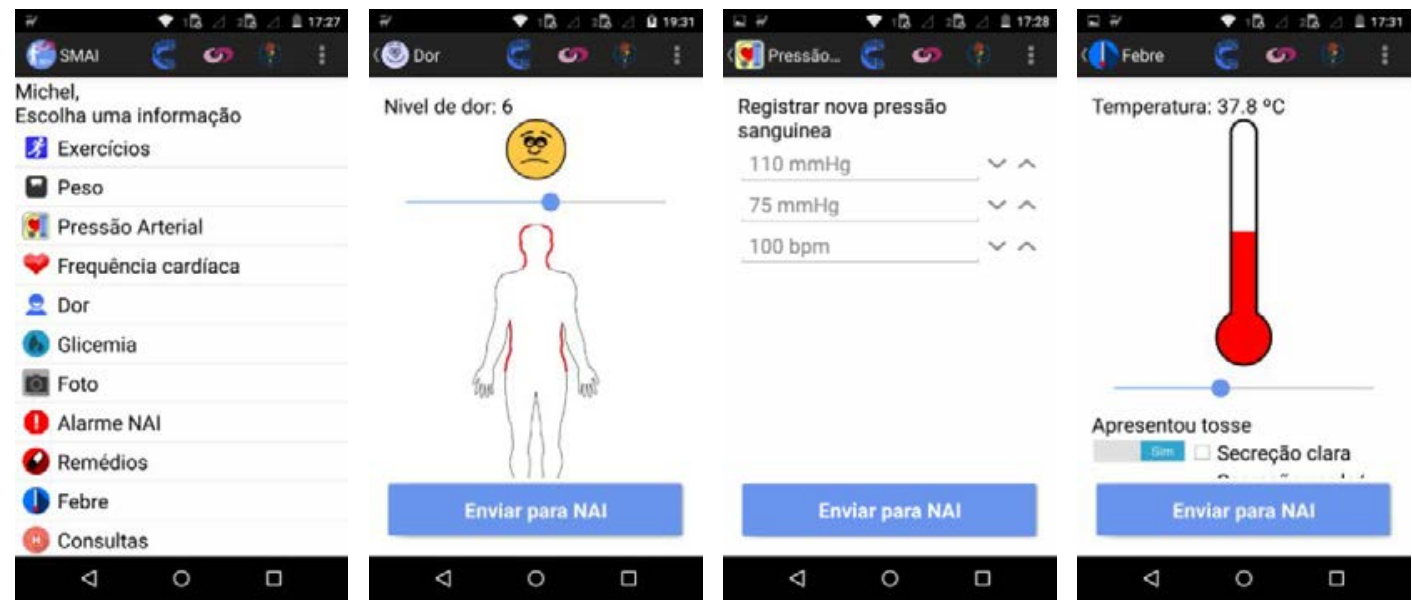

Figura 3. Tela principal e envio de informações

Diariamente, uma notificação é gerada como lembrete para o cuidador preencher um relatório (Figura 4). Ele pode preencher os itens de acordo com sua percepção de importância ou indicação do profissional de saúde. Por exemplo, na Figura 4 temos o painel de Informações Gerais, onde o cuidador anota a qualidade do sono, se houve - ou não distúrbios comportamentais e a hidratação do paciente.
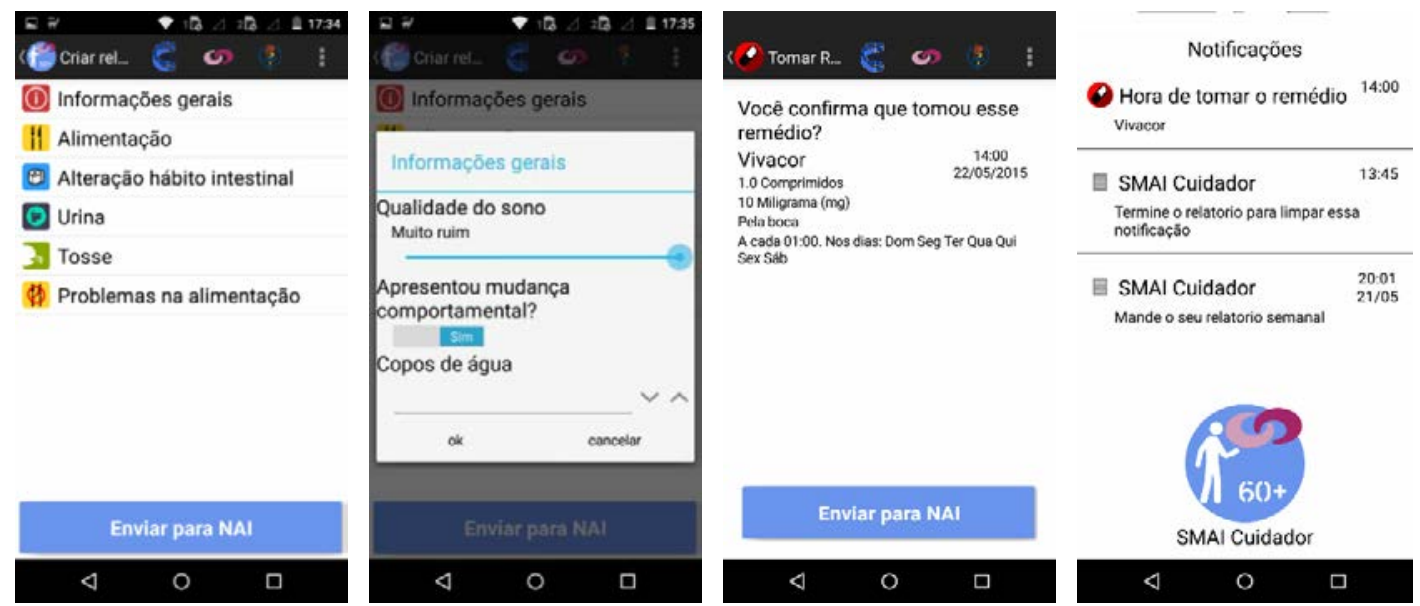

Figura 4. Relatórios, notificações e lembretes

Este é um dos módulos desenvolvidos especificamente para o conjunto de pacientes monitorados e aderentes à prática adotada pelo cuidador. Já existe uma rotina para que informações do dia a dia do paciente sejam anotadas em uma planilha em papel, de forma que a equipe de saúde possa acompanhar a evolução do paciente e intercorrências. O relatório através do SMAI objetiva tornar esta rotina mais ágil para o 
cuidador e proporcionar um acompanhamento mais próximo da equipe de saúde, já que os relatórios ficam disponíveis imediatamente.

SMAI Médico. A estrutura da aplicação do profissional de saúde se assemelha ao SMAI Cuidador (Figura 2), tendo como diferença a utilização da API de mapas da Google, além do gerenciamento de vários pacientes ao mesmo tempo.

A aplicação tem como base uma lista de pacientes monitorados e um mecanismo automático de atualização de informações que traz do serviço na nuvem as últimas informações enviadas pelos pacientes. Esta lista é persistida em um banco de dados local, e exibida no Painel de Controle inicial. Desta forma, é minimizada a necessidade de se obter os dados do servidor na nuvem a cada operação do profissional de saúde.

Pacientes ou cuidadores que acionaram o Alarme são exibidos com a borda em vermelho no Painel de Controle (Figura 5a). Quando o profissional de saúde seleciona um dos pacientes, um menu similar ao do cuidador também é apresentado (Figura 5b), com opções adicionais, como o gráfico das medidas fisiológicas transmitidas (Figura 6).
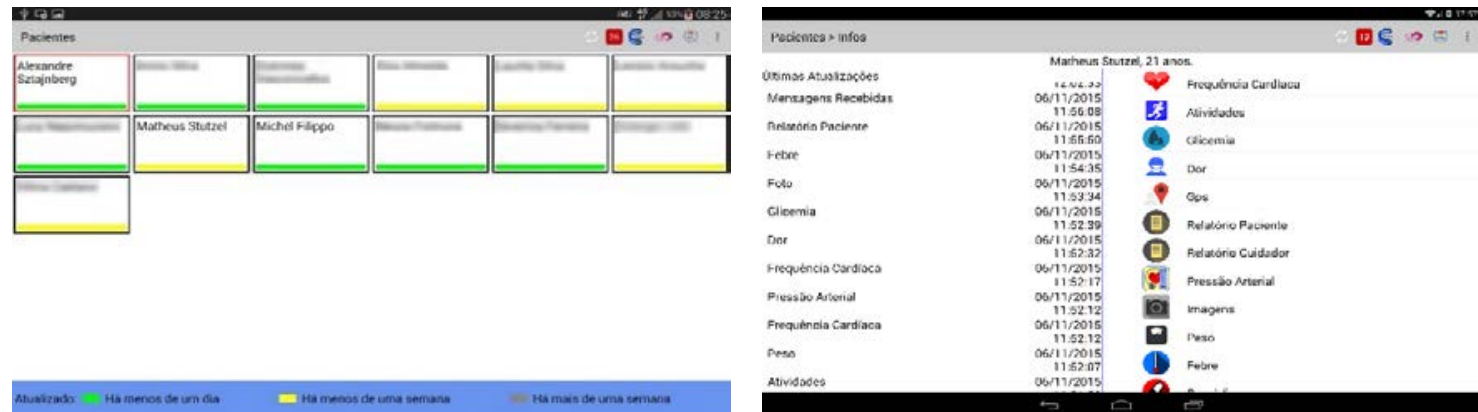

Figura 5 (a) Painel de controle, (b) detalhe de um paciente
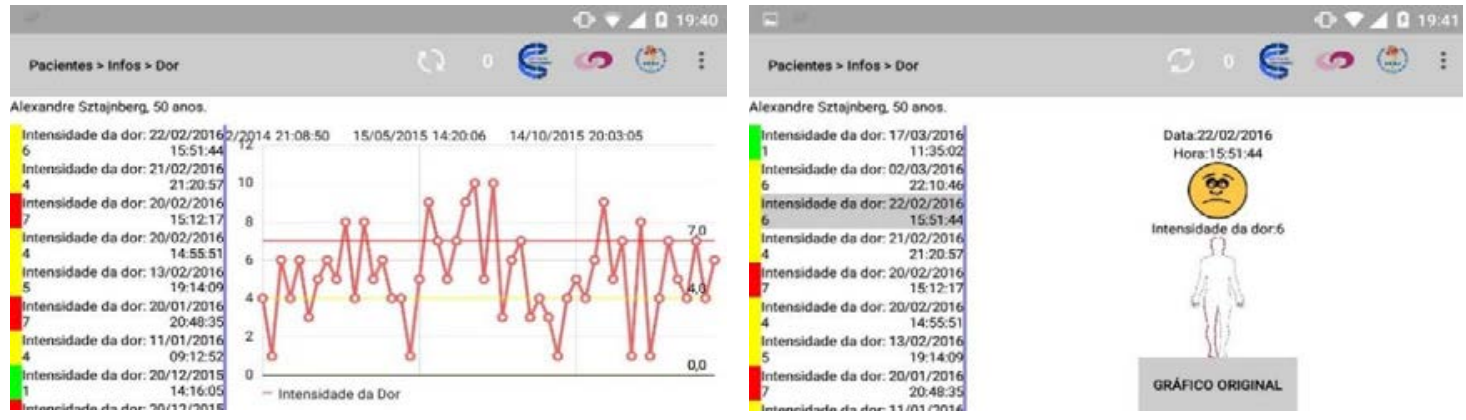

Figura 6 Detalhes do histórico de dor

Algumas opções permitem que se listem os itens enviados pelo paciente, na forma de relatórios, ou que se adicionem elementos para serem notificados para o paciente. Este é o caso dos Medicamentos, que podem ser adicionados/apagados para cada paciente. Além disso, é possível acompanhar o registro dos medicamentos que tiveram sua notificação confirmada por um cuidador. Por exemplo, o registro de localização do usuário e relatórios diários preenchidos (Figura 7) ficam à disposição da equipe de saúde para acompanhamento e tomada de decisões.

O Alarme NAI é atualizado a cada 2 minutos, com isso ele é recebido rapidamente pela equipe de saúde, tornando menor o tempo de resposta ao paciente. As demais informações também são atualizadas periodicamente de forma automática, porém em um intervalo de tempo maior. A qualquer momento podem ser solicitados os dados mais recentes, seja de um determinado paciente ou de todo o grupo. 

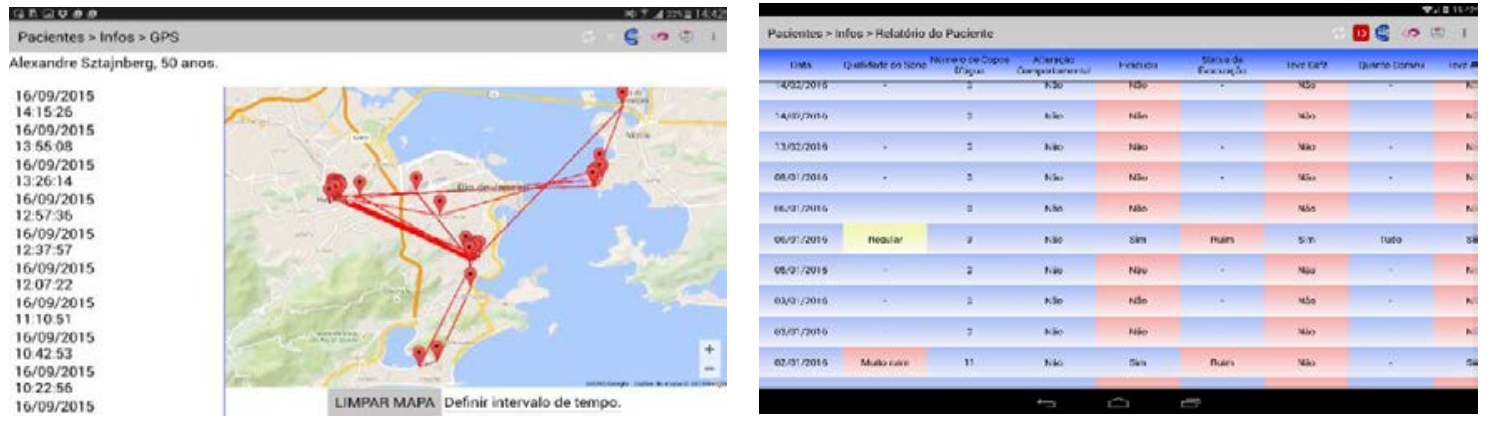

Figura 7. (a) Localização do paciente e (b) relatórios diários

\section{Avaliação}

Estudo piloto. No início de 2015 o protótipo do SMAI passou por uma primeira avaliação. Um grupo de 5 cuidadores foi selecionado. Cada um recebeu um aparelho Motorola Moto G, com o SMAI Cuidador pré-instalado e créditos suficientes. O objetivo era entender como o cuidador perceberia a nova ferramenta, facilidade de uso, intuitividade e a disposição em continuar utilizando o sistema. Para isso cada cuidador utilizaria o sistema por 15 dias, da melhor forma, sem o compromisso de fornecer dados reais. Uma reunião coletiva foi realizada para a entrega dos aparelhos e um treinamento de 40 minutos, hands-on, foi oferecido pela equipe do LCC para cada cuidador.

Durante os 15 dias da avaliação o uso do sistema foi monitorado através do SMAI Médico. Também foram monitorados os contadores de dados transmitidos e recebidos, pelo chip e pela rede WiFi (se habilitada) e qual o consumo específico da aplicação SMAI e outras aplicações. Deste monitoramento destacamos o seguinte:

- Todos os cuidadores transmitiram dados, com média variável por dia;

- O uso de dados do SMAI é baixo. Média de 87,93 kilobytes com download e de 614,04 kilobytes com upload por dia;

- As atualizações automáticas e outros mecanismos do Android representam 90\% do consumo registrado nas duas semanas de avaliação.

Durante a reunião marcada para a devolução dos aparelhos, foi realizada uma pesquisa informal de opinião com os cuidadores e as respostas foram gravadas. Foram questionados sobre a sua experiência ao usar o sistema, com destaque para os seguintes pontos: (i) todos os cuidadores fariam uso contínuo do sistema; (ii) alguns cuidadores não entenderam completamente algumas opções; (iii) sugestões foram registradas no sentido de mudar algumas opções ou acrescentar a possibilidade de informar detalhes.

Teste clínico. No final de 2015 a equipe do NAI planejou um teste clínico com o SMAI. Para isso foram desenvolvidos protocolos complementares incorporando o uso do sistema aos procedimentos e rotinas já adotados. O teste é randomizado (método de minimização) acompanhando um grupo de 30 cuidadores sob intervenção do sistema e um grupo de controle de 30 cuidadores é acompanhado com questionários por telefone. A duração prevista é de nove meses. Cada cuidador recebe um Motorola Moto $\mathrm{G}$ e um plano de dados de 50 Mbytes. Os gráficos da Figura 8 apresentam informações sobre (a) a quantidade de dados enviados, permitindo verificar quais são os módulos mais usados e (b) o consumo médio do plano de dados.

Ainda é prematuro fazer correlações e observações sobre os resultados. O consumo de dados, por exemplo, pode aumentar significativamente ao se lançar uma nova versão do 
sistema, ou quando a Google insiste em fazer atualização de alguma aplicação. Por outro lado, é possível identificar que a transmissão de dados pelos módulos iniciados a partir das notificações é maior (Relatórios, Remédios tomados).
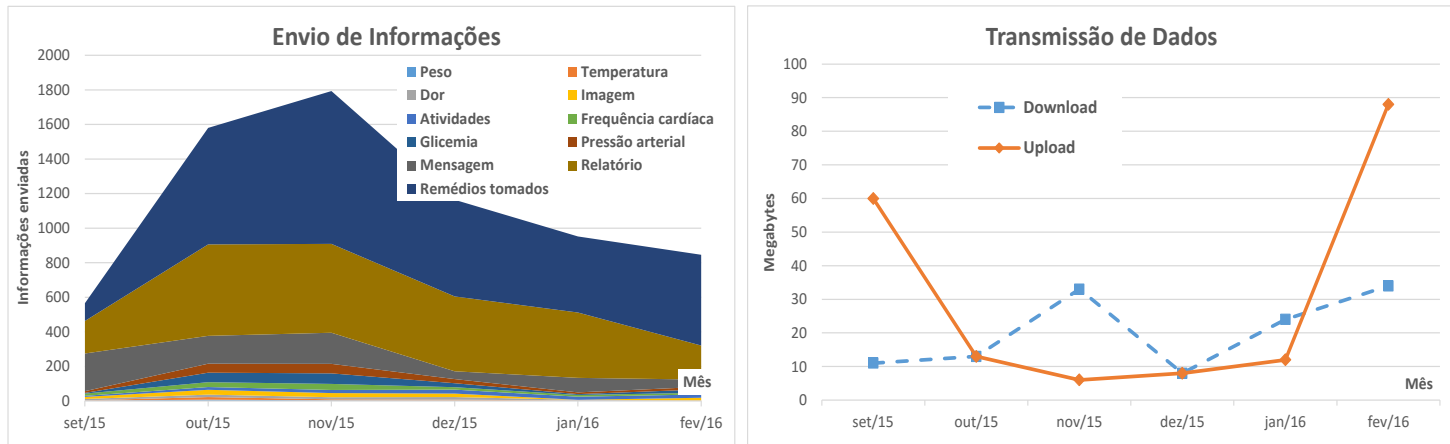

Figura 8. (a) Quantidade de informações enviadas (b) consumo de dados

O estudo tem o objetivo de avaliar a efetividade do aplicativo SMAI no monitoramento de idosos dependentes a partir da melhoria das alterações clínicas mais frequentes na população estudada. Também objetiva-se avaliar como a qualidade de vida dos cuidadores e familiares é afetada e qual a aderência dos cuidadores ao uso do sistema proposto, correlacionada aos outros aspectos avaliados.

\section{Conclusão}

A abordagem do SMAI é a simplicidade, ajudar o cuidador em sua atividade, sem ser mais uma tarefa ou preocupação, dentre tantas outras que já fazem parte do seu dia. O suporte oferecido pelo aplicativo móvel é complementar ao atendimento ambulatorial, não substituindo a avaliação clínica do paciente pela equipe de saúde.

As avaliações realizadas indicam que as opções de projeto adotadas são consistentes. O retorno dos usuários em consultas informais mostra que a usabilidade do SMAI é boa. A maioria das entradas não exige que o cuidador use o teclado virtual, mas isso não seria um problema, como verificamos em nosso levantamento preliminar. $\mathrm{O}$ desenvolvimento das aplicações, adaptados ao contexto e às possibilidades reais das pessoas envolvidas, e orientadas pelo conhecimento da equipe de saúde tem mostrado bons resultados. Além disso, a partir dos relatos de sucesso e insucesso de experimentos semelhantes na literatura [Celler, 2006], foi adotada a estratégia de tornar as aplicações atraentes ao uso, evitando o desinteresse do cuidador ou familiar.

Uma das lições aprendidas até este ponto, observado informalmente durante as reuniões presenciais com os usuários do SMAI, é o efeito de fortalecer a relação cuidador-profissional de saúde. Por exemplo, o sistema de mensagens simples do SMAI permite que a equipe NAI envie pequenos textos de orientação. Assim, além das notificações e lembretes, o cuidador pode receber da equipe NAI, mensagens personalizadas. O acompanhamento diário das informações enviadas pelos cuidadores permite que as pequenas intervenções por mensagens de texto, ou até mesmo por telefone, sejam feitas em um tempo mais curto quando comparado com procedimentos padrão. A percepção do cuidador é que o suporte é mais eficaz, o que aumenta a adesão ao uso do sistema.

Agradecimentos. Agradecemos o auxílio financeiro da FAPERJ e CNPq o apoio do InovUERJ no processo de registro do software junto ao INPI e nos pedidos de patente. 


\section{Referências}

Caldas, C. P. (2003) "Envelhecimento com dependência: responsabilidades e demandas da família", Cadernos de Saúde Pública, vol.19, no. 3, pp. 773-81.

Celler, B. G., Basilakis, J., Budge, M. e Lovel, N. H. (2006) "A clinical monitoring and management system for residential aged care facilities", 28th IEEE EMBS Annual International Conferences, pp. 3301-3304, New York, EUA.

Dorsey, E. R. (2013) "Randomized controlled trial of "virtual houses calls" for Parkinson disease", JAMA Neurology, vol. 70, no. 5, pp. 565- 70.

Finkelstein, S. M., Speedie, S. M. e Potthoff, S. (2006) "Home telehealth improves clinical outcomes at lower cost for home healthcare", Telemed. Journal and E-health, vol. 12 , no. 2 , pp. $128-38$.

Fisher, G. S. (2006) "Brief Communication: Electronic Reminds to Patiens Within an Interactive Patients", Telemed. Journal and e-Health, vol.12, no. 2, pp. 128-38.

Google, “Android”, http://www.android.com/about/

Jaglal, S.B., Haroun, V.A., Salbach, N.M., Hawker, G., Voth, J. e Lou, W. (2013) "Increasing access to chronic disease self-management programs in rural and remote communities using telehealth", Telemed. Jrnl. and e-Health, vol.19, no.6, pp.467-74.

Keranen, T. e Liikkanen, S. (2013) "Medication reminder service for mobile phones: an open feasibility study in patients with Parkinson's disease", Telemed. Journal and eHealth, vol.19, no. 11, pp. 888-90.

MinS-Ministério da Saúde, Brasil (2006) "Envelhecimento e saúde da pessoa idosa", Cadernos de Atenção Básica, n. 19. Ministério da Saúde. http://dab.saude.gov.br/ portaldab/biblioteca.php?conteudo=publicacoes/cab19

Mosa, A. S. M., Yoo, I. e Sheets, L. (2012) "A Systematic Review of Healthcare Applications for Smartphone", BioMed Central - Medical Informatics and Decision Making, vol. 12, pp. 67.

Nicholas, D., Huntington, P. e Jamali, H. (2007) Digital Health Information for the Consumer: Evidence and Policy Implications, Ashgate Publishing, Ltd., England.

Prince, M., Prina, M. e Guerchet, M. (2013) "World Alzheimer Report 2013. Journey of Caring An analysis of long-term care for dementia", Alzheimer's Disease International (ADI), London. www.alz.co.uk/worldreport2013.

Sanches, L. M., Alves, D. S., Lopes, M. H. e Novaes, M. A. (2012) "The practice of telehealth by nurses: an experience in primary healthcare in Brazil", Telemed $\mathrm{J}$ E Health, vol. 18, No 9, pp. 679-83.

Stutzel, M. C, Filippo, M. P., Sztajnberg, A., Brittes, A., Motta, L. B. (2016) "SMAI Mobile System for Elderly Monitoring", 4th IEEE SeGAH International Conference on Serious Games and Applications for Health, Orlando, EUA.

UNFPA-United Nations Population Funds (2012) "Ageing in the Twenty-First Century: A Celebration and A Challenge", Madrid. http://www.unfpa.org/webdav/site/ global/shared/documents/publications/2012/UNFPA-Exec-Summary.pdf.

Wootton, R., Craig, J. e Patterson, V. (2006) Introduction to telemedicine, Second Edition. London: The Royal Society of Medicine Press Ltd. 206p. 